\title{
Optical fiber refractometry based on multimode interference
}

\author{
Orlando Frazão, ${ }^{1, \star}$ Susana O. Silva, ${ }^{1,2}$ Jaime Viegas, ${ }^{1}$ Luís A. Ferreira, ${ }^{1}$ \\ Francisco M. Araújo, ${ }^{1}$ and José L. Santos ${ }^{1,2}$ \\ ${ }^{1}$ Instituto de Engenharia Electrónicos e Sistemas de Computadores do Porto (INESC Porto), \\ Rua do Campo Alegre 687, 4169-007 Porto, Portugal \\ ${ }^{2}$ Departamento de Física, Faculdade de Ciências da Universidade do Porto, \\ Rua do Campo Alegre 687, 4169-007 Porto, Portugal \\ ${ }^{*}$ Corresponding author: ofrazao@ inescporto.pt
}

Received 17 March 2011; revised 12 August 2011; accepted 12 August 2011; posted 12 August 2011 (Doc. ID 144236); published 19 August 2011

\begin{abstract}
This paper presents an overview of optical fiber sensors based on multimode interference with a focus on refractometric applications. A specific configuration is presented to measure the refractive index of the surrounding liquid based on the Fresnel reflection in the fiber tip, combined with a simple interrogation technique that uses two fiber Bragg gratings as discrete optical sources, with the measurand information encoded in the relative intensity variation of the reflected signals. A resolution of $1.75 \times 10^{-3} \mathrm{RIU}$ is achieved. () 2011 Optical Society of America

OCIS codes: $\quad 060.0060,060.2370,120.3180$.
\end{abstract}

\section{Introduction}

Multimode interference (MMI) in integrated optics has been extensively studied, and a variety of devices has already been developed, namely, directional couplers, adiabatic $X$ or $Y$ junctions, and diffractive star couplers [1]. In optical fiber devices, the MMI concept can also be applied by means of a step-index multimode fiber (MMF) [2]. This type of optical device can be used as wavelength filter or microbend sensor $[3,4]$. Usually, the MMI-based optical device consists of an MMF section spliced between two single-mode fibers (SMFs) forming an SMF-MMF-SMF structure (SMS). The SMS fiber structure can act as a bandpass filter or edge filter depending on the length of the MMF used [5]. Other applications are highpower fiber lasers and amplifiers based on MMI in active large-core multimode optical fibers [6], and an optical fiber-based wavelength-tunable condensing lens using this effect was also demonstrated by Mohammed et al. [7].

0003-6935/11/25E184-05\$15.00/0

(C) 2011 Optical Society of America
The MMI optical fiber device has increasingly been considered of high potential for sensing considering the performance possible to be achieved, namely, in refractive-index (RI) measurement. This work addresses this topic, starting by presenting a short overview of the subject and then focusing on utilization of this structure as a refractometer. A particular refractometric configuration is then researched, and a simple interrogation technique is proposed compatible with readout $\mathrm{RI}$ resolutions adequate for a large range of applications.

\section{Historic Overview of Multimode-Interference-Based Fiber Sensing}

The first sensor based on MMI using a multimode step-index fiber was studied in 2003 by Mehta et al. [8]. The theoretical model and experimental setup of a displacement sensor were demonstrated. The structure consisted of splicing an MMF section between two SMFs. The end of the MMF was cleaved and combined with a planar mirror to form the displacement sensor. In 2006, Jung et al. [9] proposed a compact surrounding RI sensor using an MMFcoreless-silica-fiber-MMF-based structure where the 
evanescent waves in the coreless silica fiber (CSF) region could directly interact with the surrounding medium. The suggested device has shown high potential for a wide range of biomedical and chemical sensor applications. In the same year, $\mathrm{Li}$ et al. [10] developed a fiber-optic temperature sensor in reflection that relied on the interference between selective higher order modes in multimode optical fibers. It was demonstrated that by coupling the $\mathrm{LP}_{01}$ mode in a standard SMF to the $\mathrm{LP}_{0 m}$ modes in an MMF, and utilizing the interference of the higher order modes, a fiber-optic high-temperature sensor could be obtained. In another perspective, Mohammed et al. [11] reported a fixed-wavelength bandpass filter based on MMI and the reimaging concept. Various factors were investigated, such as MMF length and geometries of the SMF and MMF that affected the filter center wavelength, bandwidth, and isolation. It was shown that the bandwidth of the device changed when the strain was applied. Later, Wang and Farrell [12] employed a modeling tool for simulation and design of a similar structure as an optical fiber refractometer sensor. In 2007, Frazão et al. [13] demonstrated an all-fiber Mach-Zehnder interferometer based on an MMI-based fiber structure combined with a long-period fiber grating (LPG) for the measurement of curvature. In 2009, Zhang et al. [14] studied a sensing scheme to measure temperature and strain simultaneously by multiplexing a section of an MMF and a Fizeau etalon interferometer. Also in the same year, Tripathi et al. [15] presented a detailed study of the strain and temperature-sensing characteristics of SMS fiber structures based on a graded-index MMF section spliced between two SMFs. In 2010, Zhang and Peng [16] reported a refractometer based on an SMS fiber structure. The $\mathrm{HF}$ corrosion method was also applied in order to improve the sensitivity of the sensing device. Recently, a simple displacement sensor based on a bent SMS fiber structure was experimentally investigated by Wu et al. [17]. Moreover, Silva et al. [18] characterized an SMS structure based on a large-core, air-clad photonic crystal fiber (PCF) as a refractometer sensor. Using two distinct large-core air-clad PCF geometries, one for RI measurement and the other for temperature compensation, it was possible to implement a sensing head that was sensitive to RI changes induced by temperature variations in water. In another perspective, a temperature- and strainindependent curvature sensor was attained by means of the SMS fiber structure technology [19]. Later, Wang et al. [20] proposed an evanescent field fiber refractometer based on a tapered MMF sandwiched between two SMFs, and Wu et al. [21,22] reported two works for measurement of the RI based on the combination of an SMS fiber structure followed by a fiber Bragg grating (FBG) with different core sizes in the MMF. Table 1 presents an overview of the various types of MMI-based fiber-optic structures that already have been developed.
Table 1. MMI-Based Optical Fiber Sensors

\begin{tabular}{llc}
\hline Structure Device & \multicolumn{1}{c}{ Sensor Type } & Reference \\
\hline SMF-MMF/mirror & Displacement & {$[\underline{8}]$} \\
MMF-CSF-MMF & Refractometer & {$[\underline{9}]$} \\
SMF-MMF & High-temperature & {$[\underline{10}]$} \\
SMF-MMF-SMF & Strain & {$[\underline{11}]$} \\
SMF-MMF-SMF & Refractometer & {$[\underline{12}]$} \\
SMF-MMF/LPG & Curvature & {$[\underline{13}]$} \\
SMF-MMF/Fizeau & Simultaneous measurement & {$[\underline{14}]$} \\
& of strain and temperature & \\
SMF-graded-index & Strain and temperature & {$[\underline{15}]$} \\
MMF-SMF & Refractometer & {$[\underline{16}]$} \\
SMF-etched & Displacement and bent & {$[\underline{17}]$} \\
MMF-SMF & Refractometer & {$[\underline{18}]$} \\
SMF-MMF-SMF & Temperature and strain & {$[\underline{19}]$} \\
SMF-LC-air-clad & independent curvature & \\
PCF-SMF & sensor & {$[\underline{20}]$} \\
SMF-MMF-SMF & Refractometer & {$[\underline{21}]$} \\
& &
\end{tabular}

\section{Optical Fiber Refractometry Based on Multimode Interference}

In the past few years, many efforts have been devoted to optical fiber-based-refractometer sensors, which have shown to be a promising and attractive technology in chemical and biotechnological applications. MMI-based devices for RI sensing using multimode optical fibers have been comprehensively studied due to their high spectral selectivity, high sensitivity, and ease of fabrication. A simple scheme proposed by Jung et al. [9] made use of evanescent wave absorption in a $\mathrm{CSF}$ section spliced between two MMFs. The sensing concept was based on the evanescent wave interaction between the CSF and the surrounding medium. For the operation wavelength at $1300 \mathrm{~nm}$ and a CSF length of $34 \mathrm{~mm}$, the $\mathrm{RI}$ resolution achieved was $4.37 \times 10^{-4} \mathrm{RIU}$ for the RI range from 1.3 to 1.44. Wang and Farrell [12] presented instead a numerical simulation for an optical fiber refractometer based on MMI-the sensing structure was an MMF core section spliced between two SMFs where the surrounding liquid sample worked as the cladding medium. For a different $\mathrm{RI}$, interference between different modes will occur while the light propagates along the MMF core section. For operation at a wavelength of $1550 \mathrm{~nm}$ and an optimized MMF length of $9.42 \mathrm{~mm}$, the RI resolution was estimated to be $5.4 \times 10^{-5}$ RIU for an RI range from 1.33 to 1.45. Later, Zhang and Peng [16] demonstrated experimentally the feasibility of an SMS fiber structure as a refractometer sensor where an MMF section with different HF-corroded cladding diameters was used. Results showed that the resolution could be improved by using an MMF core with full HF-corroded cladding. For the operation wavelength at $1550 \mathrm{~nm}$ and an MMF length of $48 \mathrm{~mm}$, the 
$\mathrm{RI}$ resolution achieved was $7.9 \times 10^{-5} \mathrm{RIU}$ for an $\mathrm{RI}$ range from 1.33 to 1.38. Recently, an SMS structure based on a large-core, air-clad PCF was reported by Silva et al. [18]. The sensing head was based on multimodal interference, and it relied on a singlemode/large-core air-clad PCF/SMF configuration. Two distinct large-core air-clad PCF geometries were used, one for RI measurement and the other for temperature compensation, with fiber lengths of 4.2 and $4.3 \mathrm{~cm}$, respectively. This sensing head allowed measuring RI changes induced by temperature variations in water, and a resolution of $3.4 \times 10^{-5} \mathrm{RIU}$ could be achieved. Later, Wang et al. [20] proposed and experimentally demonstrated an evanescent field fiber refractometer based on a tapered MMF sandwiched between two SMFs. A maximum sensitivity of $1913 \mathrm{~nm} / \mathrm{RIU}$ was achieved with a $\sim 30 \mu \mathrm{m}$ MMF taper waist diameter. Also, Wu et al. [21] reported an RI sensor based on the combination of an SMS fiber structure followed by an FBG. In the same line of research, the same group [22] investigated the influence of MMF core diameters and lengths on the sensitivity of an SMS-fiber-based refractometer. They have shown that the MMF diameter had significant influence on the RI sensitivity but no dependence on the fiber length.

The readout instrument used to interrogate the MMI-based fiber-optic structures was always an optical spectrum analyzer (OSA), regardless of the configurations described previously to obtain RI information. In the following section, a simple interrogation technique is presented for RI measurement applied to an MMI-based fiber tip structure. The interrogation technique uses two FBGs as discrete optical sources that, by means of relative intensity variation of the reflected signals, will provide measurement of RI changes while using the advantages of the MMI-based fiber tip layout.

\section{Experimental Interrogation System}

The experiment described addresses a sensing configuration that combines an optical fiber tip based on the MMI concept with Fresnel reflection at the fiber-to-liquid interface with a versatile and efficient FBG-based interrogation technique. The testing procedure involves inserting the fiber tip into liquids possessing a distinct RI. Fresnel reflection obtained from the fiber-to-liquid interface will thus be expected to change according to the actual RI.

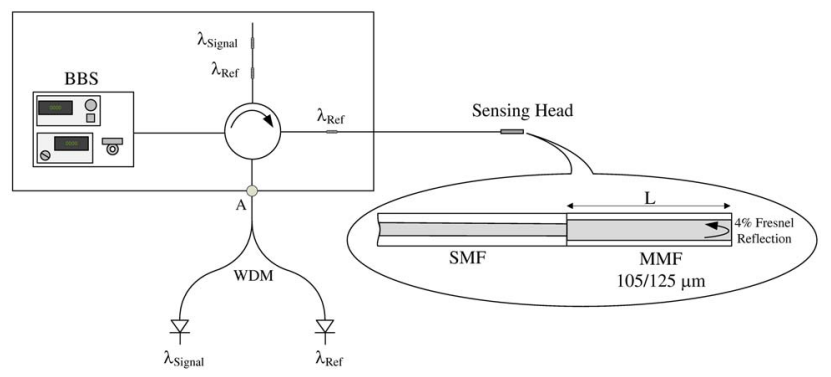

Fig. 1. Experimental setup of the interrogation system.
To demonstrate the proposed configuration, the sensing-head structure shown in Fig. 1 was implemented. To obtain the band reject filter in the region of $1550 \mathrm{~nm}$, the length of the sensing head is important. In the literature, the length is approximately $50 \mathrm{~mm}$ [10]. However, in this case, the length is independent of the external physical parameter. The sensing structure consists of a $47 \mathrm{~mm}$ long step-index MMF (with a numerical aperture of 0.22 and core and cladding diameters of 105 and $125 \mu \mathrm{m}$, respectively), spliced at the end of an SMF and interrogated in reflection. The end of the MMF was then cleaved in order to obtain Fresnel reflection at the fiber tip.

Before interrogating the sensing head, the optical spectrum was observed by means of an OSAFig. 2-placed on the last output port of the optical circulator (point $A$ in Fig. 1). Figure 2 shows the location of the Bragg gratings to interrogate in reflection the MMI structure.

The interrogation system uses a broadband source (central wavelength at $1550 \mathrm{~nm}$ and bandwidth of $100 \mathrm{~nm}$ ) connected to a four-port optical circulator that, in turn, illuminates two FBGs in the second output port. The purpose is to reflect two discrete narrow optical sources with different wavelength resonances: one is then used as the reference and the other as the signal (see Fig. 3). In output port three, the MMI-based fiber tip structure is placed, together with a second FBG with the same wavelength of the reference signal, in order to eliminate broadband source fluctuations and losses along the fiber. On the last output port of the optical circulator, a WDM device was placed-with the purpose of separating both FBG wavelengths-and two photodetectors to read the optical power of each FBG signal.

The optical spectrum of the MMI-based fiber tip presents a wide passband, delimited by two welldefined loss peaks, located at resonances of 1532.5 and $1546.1 \mathrm{~nm}$ and separated by $13.6 \mathrm{~nm}$. When the light field propagating along the input SMF enters the MMF section, high-order modes of MMF are

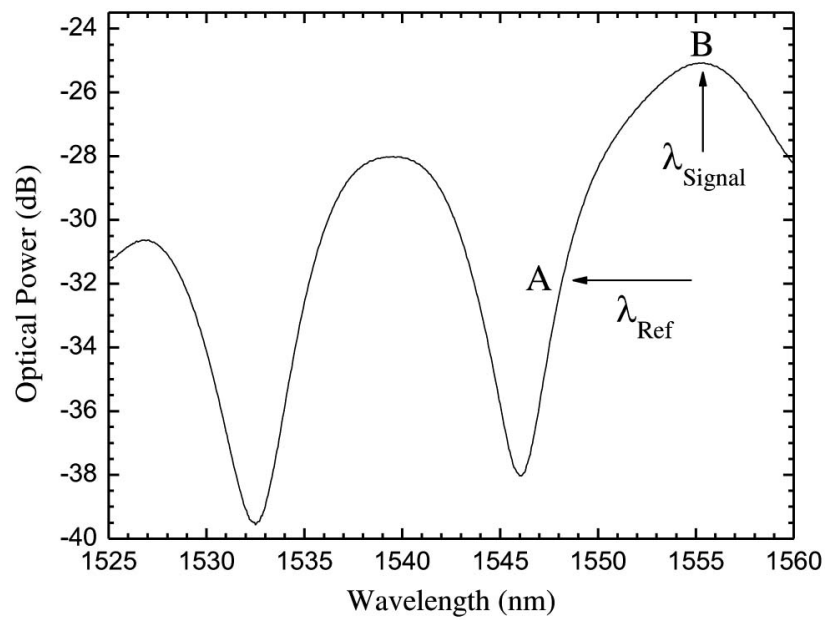

Fig. 2. Spectral response of the MMI structure in reflection. 


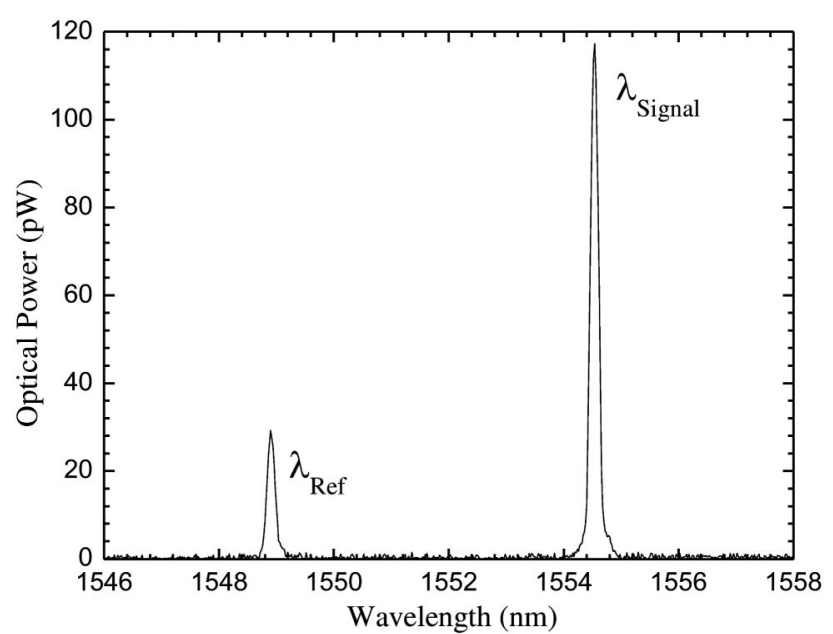

Fig. 3. Two discrete narrow sources based on FBGs.

excited; hence, interference between different modes will occur, while the light beam propagates along the remainder of the MMF section. The loss peaks shown in Fig. 2 are therefore produced by destructive interference between two specific higher order modes, $\mathrm{LP}_{0 m}$ and $\mathrm{LP}_{0(m-1)}$, at the exit end of the MMF.

The optical power along the MMF section varies periodically with the wavelength and the wavelength spacing between two adjacent maxima (or minima), $m$ and $(m-1)$, is predicted by $[\underline{10]}$

$$
\Delta \lambda=\frac{16 n_{\mathrm{co}} a^{2}}{(4 m-3) L},
$$

where $a$ is the core radius of the MMF, $n_{\text {co }}$ is the RI of the MMF core, and $L$ is the MMF length in reflection. In this case, it was estimated that destructive interference was produced between the $\mathrm{LP}_{012}$ and $\mathrm{LP}_{013}$ modes in the MMF. To confirm this, the wavelength spacing was calculated using the following parameters: $L=94 \mathrm{~mm}$ (twice the MMF length), $m=13, a=52.5 \mu \mathrm{m}$, and $n_{\text {со }}=1.4662$. Therefore, the spacing between the various resonances is estimated as $\sim 14 \mathrm{~nm}$, which is in close proximity of the measured value $(13.6 \mathrm{~nm})$.

To perform the experiment, two FBGs with distinct resonance wavelengths were used, as depicted in Fig. 2. One FBG is used as the reference $\left(\lambda_{\text {Ref }}\right)$ and the other as the signal $\left(\lambda_{\text {Signal }}\right)$, with resonances at (1548.9) and (1554.54) nm, respectively. The FBGs were specifically designed to be spectrally located at points of the MMI-based fiber tip optical spectrum with different RI sensitivities-as marked by $A$ and $B$ in Fig. 2. If the optical power of $\lambda_{\text {Ref }}$ and $\lambda_{\text {Signal }}$ that photodetectors detect is $P_{\text {Ref }}$ and $P_{\text {Signal }}$, respectively, then processing of the type $\left(P_{\text {Signal }}-P_{\text {Ref }}\right) /\left(P_{\text {Signal }}+\right.$ $\left.P_{\text {Ref }}\right)$ gives a signal proportional to the measurandinduced visibility changes of the MMI interferometer, i.e., independent of the optical power fluctuations along the system.

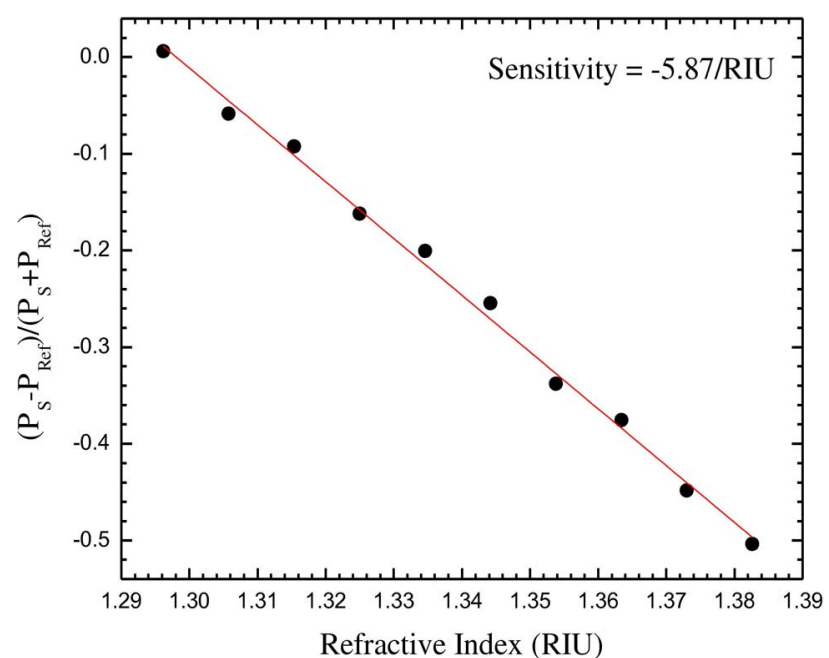

Fig. 4. (Color online) RI measurement using the experimental setup of Fig. $\underline{1}$.

The behavior of this structure as an RI sensor was duly characterized. The sensing-head characterization was performed by placing the sensing structure in contact with liquids with distinct RIs in the range 1.30-1.38; a series of commercial RI standards from Cargille Laboratories (Cedar Grove, N.J.) were accordingly used; such standards were properly corrected for wavelength and temperature. From the registered $P_{\text {Ref }}$ and $P_{\text {Signal }}$ values, the data shown in Fig. 4 were obtained, indicating an RI sensitivity of $-5.87 /$ RIU.

To ascertain the system resolution to RI variations, the signal change associated with a step change of $0.01 \mathrm{RIU}$ was measured; the results obtained are shown in Fig. 5. Based on the step changes and rms fluctuations, one could calculate an RI resolution of $1.75 \times 10^{-3} \mathrm{RIU}$.

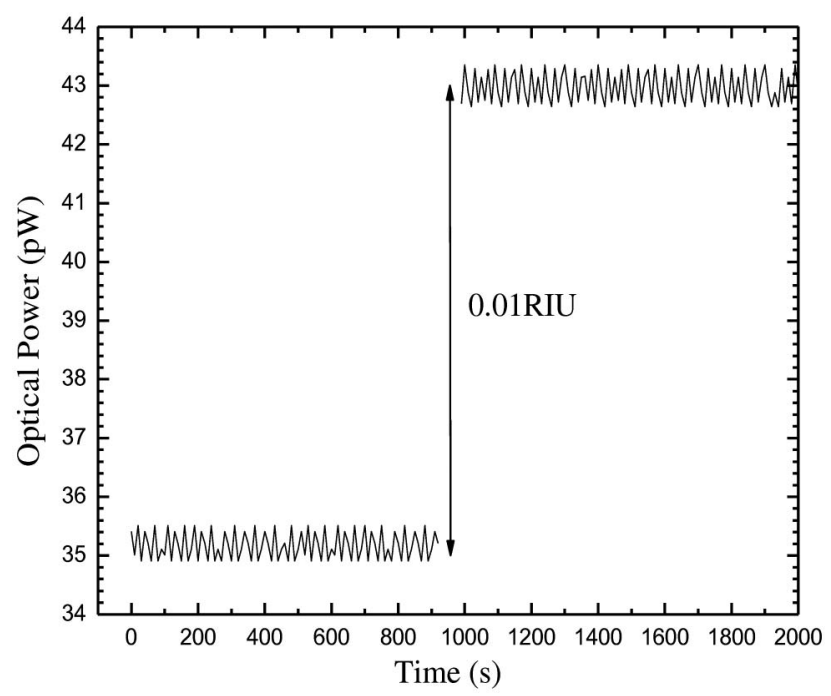

Fig. 5. Determination of the RI resolution obtained with the experimental setup of Fig. 1 . 
This value can be improved by selecting a pair of FBGs with an optimum spectral position relatively to the interferometric MMI channeled spectrum. Anyway, this performance is good enough for a large range of applications involving RI measurement, particularly if the small size of the sensing probe (effectively a fiber tip) is considered, allowing highly localized sensing (in remote regions if necessary), and the intrinsic simplicity of the interrogation approach. It is also worthwhile to mention that the MMI structure shown in Fig. 1 with a certain degree of etching of the multimode section will turn the interferometric phase sensitive to RI variations of the external medium, which means much higher sensitivities would become feasible applying processing techniques that have been developed along the years to interrogate fiber-optic interferometric sensors.

\section{Conclusions}

This work presented an overview of fiber-optic sensors based on the MMI concept with a focus on the measurement of the RI. It was realized that invariably an OSA has been used to read out the sensing signal, which turns out to be a serious limitation for the application of fiber-optic sensing structures based on this principle. In view of this, the feasibility of a simple interrogation technique relying on the utilization of FBGs was demonstrated, reporting results that indicate performance compatible with a large range of applications.

\section{References}

1. L. B. Soldano and E. C. M. Pennings, "Optical multi-mode interference devices based on self-imaging: principles and applications," J. Lightwave Technol. 13, 615-627 (1995).

2. A. Kumar, R. K. Varshney, C. S. Antony, and P. Sharma, "Transmission characteristics of SMS fiber optic sensor structures," Opt. Commun. 219, 215-219 (2003).

3. Q. Wang and G. Farrell, "Multimode fiber based edge filter for optical wavelength measurement application," Microw. Opt. Technol. Lett. 48, 900-902 (2006).

4. D. Donlagic and M. Zavrsnik, "Fiber-optic microbend sensor structure," Opt. Lett. 22, 837-839 (1997).

5. Q. Wang, G. Farrell, and W. Yan, "Investigation on single mode-multimode-single mode fiber structure," J. Lightwave Technol. 26, 512-519 (2008).

6. X. Zhu, A. Schulzgen, H. Li, L. Li, V. L. Temyanko, J. V. Moloney, and N. Peyghambarian, "High-power fiber lasers and amplifiers based on multimode interference," IEEE J. Sel. Top. Quantum Electron. 15, 71-78 (2009).

7. W. S. Mohammed, A. Mehta, and E. G. Johnson, "Wavelength tunable fiber lens based on multimode interference," J. Lightwave Technol. 22, 469-477 (2004).
8. A. Mehta, W. S. Mohammed, and E. G. Johnson, "Multimode interference-based fiber-optic displacement sensor," IEEE Photon. Technol. Lett. 15, 1129-1131 (2003).

9. Y. Jung, S. Kim, D. Lee, and K. Oh, "Compact three segmented multimode fibre modal interferometer for high sensitivity refractive-index measurement," Meas. Sci. Technol. 17, 1129-1133 (2006).

10. E. Li, X. Wang, and C. Zhang, "Fiber-optic temperature sensor based on interference of selective higher-order modes," Appl. Phys. Lett. 89, 091119 (2006).

11. W. S. Mohammed, P. W. E. Smith, and X. Gu, "All-fiber multimode interference bandpass filter," Opt. Lett. 31, 2547-2549 (2006).

12. Q. Wang and G. Farrell, "All-fiber multimode-interference based refractometer sensor: proposal and design," Opt. Lett. 31, 317-319 (2006).

13. O. Frazão, J. Viegas, P. Caldas, J. L. Santos, F. M. Araújo, L. A. Ferreira, and F. Farahi, "All-fiber Mach-Zehnder curvature sensor based on multimode interference combined with a long-period grating," Opt. Lett. 32, 3074-3076 (2007).

14. J. Zhang, Y. Zhang, W. Sun, and L. Yuan, "Multiplexing multimode fiber and Fizeau etalon: a simultaneous measurement scheme of temperature and strain," Meas. Sci. Technol. 20, 065206 (2009).

15. S. M. Tripathi, A. Kumar, R. K. Varshney, Y. B. P. Kumar, E. Marin, and J.-P. Meunier, "Strain and temperature sensing characteristics of single-mode-multimode-singlemode structures," J. Lightwave Technol. 27, 2348-2356 (2009).

16. J. Zhang and S. Peng, "A compact SMS refractometer based on HF corrosion scheme," presented at the Symposium on Photonics and Optoelectronic (SOPO), Chengdu, China, June 19-21 2010, pp. 1-4.

17. Q. Wu, Y. Semenova, P. Wang, A. M. Hatta, and G. Farrell, "Experimental demonstration of a simple displacement sensor based on a bent single mode-multimode-single mode fiber structure," Meas. Sci. Technol. 22, 025203 (2011).

18. S. Silva, J. L. Santos, F. X. Malcata, J. Kobelke, K. Schuster, and O. Frazão, "Optical refractometer based on large-core air-clad photonic crystal fibers,” Opt. Lett. 36, 852-854 (2011).

19. S. Silva, O. Frazão, J. Viegas, L. A. Ferreira, F. M. Araújo, F. X. Malcata, and J. L. Santos, "Temperature and strainindependent curvature sensor based on a singlemode/ multimode fiber optic structure," Meas. Sci. Technol. 22, 085201 (2011).

20. P. Wang, G. Brambilla, M. Ding, Y. Semenova, Q. Wu, and G. Farrell, "High-sensitivity, evanescent field refractometric sensor based on a tapered, multimode fiber interference," Opt. Lett. 36, 2233-2235 (2011).

21. Q. Wu, S. B. Yan, Y. Ma, P. Wang, C. Yu, and G. Farrell, "Fiber refractometer based on a fiber Bragg grating and singlemode-multimode-single-mode fiber structure," Opt. Lett. 36, 2197-2199 (2011).

22. Q. Wu, Y. Semenova, P. Wang, and G. Farrell, "High sensitivity SMS fiber structure based refractometer-analysis and experiment," Opt. Express 19, 7937-7944 (2011). 